\title{
Multiobjective Improved Particle Swarm Optimisation for Transmission Congestion and Voltage Profile Management using Multilevel UPFC
}

Research Article

\author{
Mallavolu Malleswara Rao' ${ }^{1}$ Dr. Geetha Ramadas²
}

${ }^{1}$ Mallavolu Malleswara Rao, Research Scholar, Anna university, Chennai, India.

${ }^{2}$ Dr. Geetha Ramads, Professor \& Hod EEE, RMK Engineering College, Chennai, India

Received December 13, 2018; Accepted February 09, 2019

Abstract: This paper proposes a multiobjective improved particle swarm optimisation (IPSO) for placing and sizing the series modular multilevel converter-based unified power flow controller (MMC-UPFC) FACTS devices to manage the transmission congestion and voltage profile in deregulated electricity markets. The proposed multiobjective IPSO algorithm is perfect for accomplishing the close ideal distributed generation (DG) sizes while conveying smooth assembly qualities contrasted with another existing algorithm. It tends to be reasoned that voltage profile and genuine power misfortunes have generous upgrades along ideal speculation on DGs in both the test frameworks. The proposed system eliminates the congestion and the power system can be easily used to solve complex and non-linear optimisation problems in a real-time manner.

Keywords: Congestion • Unified power flow controller • Improved particle swarm optimisation • Modular multilevel converter • voltage profile

\section{Introduction}

In 1992, Gyugi introduced unified power flow controller (UPFC) to compensate for both the series and shunt compensation in the transmission line (Gyugyi, 2003). The existing FACTS device controls only one power at a time, that is, active or reactive power but the most powerful UPFC FACTS regulate both the power at the same time of a single transmission line. In order to trigger the application of UPFC, some extra regulatory freedoms are required to compensate the problem of congestion and voltage profile.

The basic configuration of conventional UPFC with two parallel and serial voltage source inverters is shown in Fig. 1. Connect the two inverters through a common dc link. UPFC has the ability to regulate the simultaneous or selective flow of power transmission by controlling the angle of the voltage generated by the series converter. The existing UPFC can share the dc link commonly for both inverters; the transformer acts as an interface between the inverters and the transmission line and exchange the active power between them. In order to integrate both the inverters, Zigzag transformers are required. But these transformers are of high volume, cost, and power loss of more than $50 \%$. In recent days, the multilevel inverters are introduced to overcome the drawback present in the Zigzag transformers. Voltage source converter (VSC) exists in the UPFC, but it achieves only poor output performance due to the restricted voltage and current ratings of the switching devices.

Recently, modular multilevel converter (MMC) overcomes all the other inverters because of its modular cascaded structure. It has the capacity to increase the quality of the output waveform. Such converters were mainly suitable for the high voltage and large capacity applications (Guan and Xu, 2012; Tu et al., 2011). This MMC-UPFC project was first launched by China in the year 2015 in Nanjing. MMC-based UPFC inspires the researchers of the power industry, and some projects in the same MMC-based UPFC with more flexible and high 


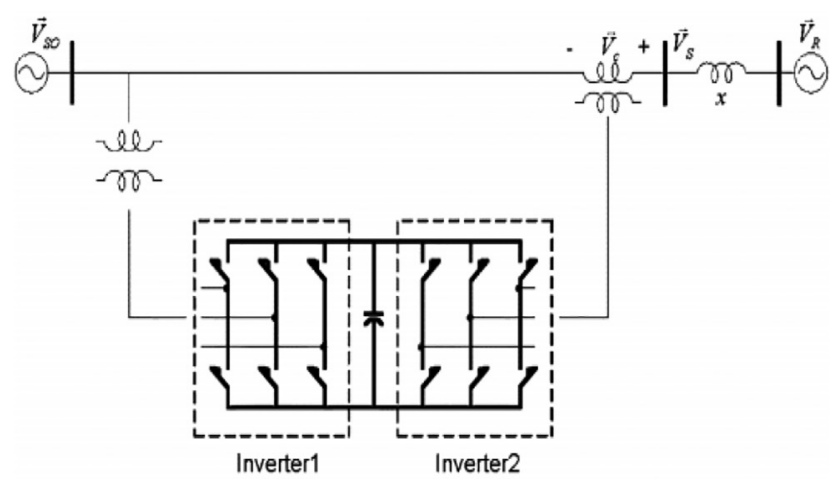

Fig. 1. Conventional configuration of unified power flow controller (Wang and Peng, 2004)

rating are under construction. The MMC-UPFC FACTS placement of optimal and the sizing manage the problem of congestion. Electric power is the major demand even though the power generation has increased more than ever before (Yamin and Shahidehpour, 2003). The Transcos, Genesis, and Discos power companies were not able to achieve the reliability of power supply due to the high demand for electricity, which leads to the congestion fundamental deregulated environment problem.

Congestion may occur in the network lines due to the unexpected load increase, lower thermal limits, and line outages (Pillay et al., 2015). In the network corridor, a sudden increase in the flow of power also leads to congestion by a mixture of two transactions, that is, bilateral and multilateral. Many researchers have tried several methods for reducing electricity flow in network lines or congestion management of a large-scale power system in the past decades (Kumar et al., 2005). Increase in three major factors such as operating costs, transient stabilities, and voltage lead to congestion. So, the congestion management problem can be solved by optimal sizing and placing of FACTS devices with the consideration of the abovementioned factors as objectives (Kumar and Sekhar, 2013).

Particle swarm optimisation (PSO; Dutta and Singh, 2008; Hajforoosh et al., 2012; Jordehi, 2015) is one of the optimisation algorithms to solve the problem of congestion management. Also, some other techniques such as Fuzzy Adaptive Bacterial Foraging Algorithm (F-ABFA) (Venkaiah and Vinod Kumar, 2011) and its advanced form of ABFA with Nelder-Mead (ABFA-NM) (Panigrahi and Ravikumar Pandi, 2009) are better-suited testing in a network of the bulk power systems. The algorithms such as Ant Lion Optimiser (ALO) (Mukherjee and Verma, 2016) and the Firefly Algorithm (FA) (Mishra and Kumar Gundavarapu, 2016) also provide support to the same problem.

The significant contribution of the paper is as follows:

- The investigation of MMC-UPFC under the multiobjective proposed control scheme by mathematical derivation.

- Streamlining the requirements of disparity to accomplish ideal measuring of distributed generation (DG) units.

- Combining the various targets into a single goal so as to accomplish the most ideal sizes of the DGs.

The remaining sections of the manuscript are described as follows. In Section 2, the works relevant to our approach are discussed. Section 3 investigates the modelling of the MMC-UPFC. The problem to be considered is described in Section 4. The DG optimal sizing with multiobjective function is defined in Section 5. The novel algorithm is described in Section 6, and Section 7 provides the result and analysis of the proposed approach. Finally, Section 8 concludes the work with its advantages.

\section{Related work}

Some of the recent related works are discussed as follows:

Numerous approaches have been developed to avoid the problems of conventional UPFC controllers. Albatsh et al. (2017) have used the fuzzy logic controller (FLC) to deliver the control of dynamic power via lines of transmission. 
The FLC offers faster response than the conventional PI controller, and it does not need mathematical precise modelling during its dynamic operations. Their proposed controller overcomes the drawbacks of conventional UPFC topology by using a transformerless converter and multilevel inverter-based UPFC.

Raj and Bhattacharyya (2018) have applied four dissimilar algorithms to obtain the optimal setting of all different compensators by control variables. The optimisation algorithms such as the Quasi opposition-based Grey wolf optimisation (QOGWO), Quasi opposition-based Differential Evolution (QODE), Grey wolf optimisation (GWO) differential evolution (DE), and the Whale optimisation algorithm (WOA) are used to identify the optimal control variables of FACTS device in the test system. The optimal control variables for series and the shunt type of compensator include Thyristor Controlled Series Compensator (TCSC) and Static Var compensator (SVC) to minimise the cost of operation and the system active power loss.

Batra and Ghosh (2018) have presented hybrid optimisation criteria to solve the problem of non-linear congestion management. They presented an algorithm optimised for the optimisation of the chaotic particles swarm (ITMCPSO) embedded in the tent map, which was the combination of PSO with optimisation of a swarm of adaptive chaotic particles of tent maps to prevent local minima in the case of non-linear problems. The search procedure of the proposed ITM-CPSO algorithm chooses the optimisation variables within a normalised search space to solve the congestion management problem.

Moreover, Kapse et al. (2018) have also proposed a hybrid combination of the optimisation algorithm to solve the problem of optimal reactive power dispatch. The hybrid algorithm is the concatenation of the Genetic algorithm and PSO algorithm to improve power transfer capability and reduce network loss. It also controls the voltage for dependent and independent control variables at various buses. UPFC is the FACTS device used for their analysis using reactive power injection to enable the reactive power injection. The placement of the UPFC was the most important challenge taken for their research. For the analysis, the two-voltage source model has been used. Their proposed hybrid optimisation approach optimally selects the control parameters of the UPFC controller for calculating the reactive power dispatch optimally. They tested and obtained the results initially by applying GA and PSO algorithms individually. After that, they tried with the combination of both GA and PSO.

\section{MMC-UPFC modelling and formulation}

Fig. 2 shows the basic configuration of MMC-UPFC, which consists of a shunt MMC and a series one connected back to back. The shunt MMC usually provides the stiff dc link voltage and also controls the shunt reactive power to support the ac bus voltage. The series one generates one controllable ac voltage and couples it to the transmission line through the series transformer, to regulate the power flow of the line. Thyristor bypass switch (TBS) and mechanical bypass switch (MBS) are used to bypass MMC-UPFC temporarily and for a long time, respectively.

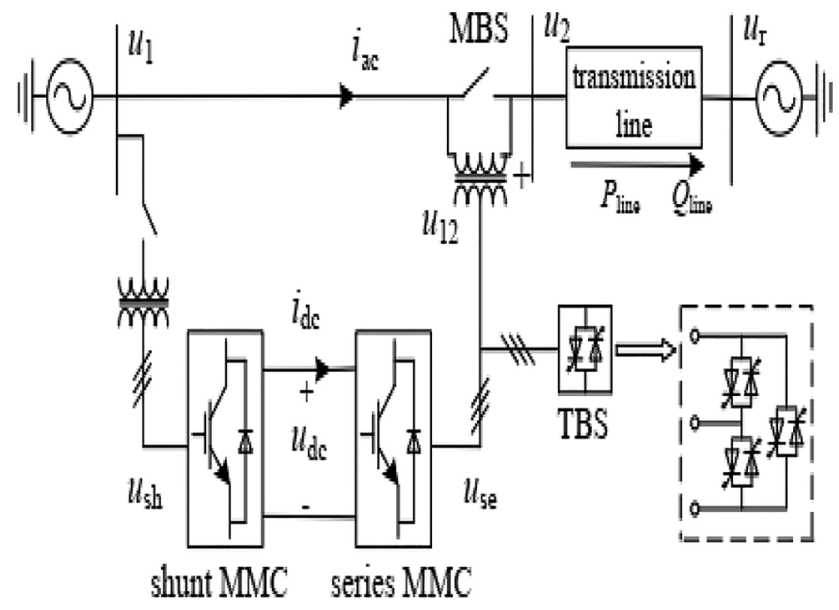

Fig. 2. Configuration of modular multilevel converter-based unified power flow controller (MMC-UPFC) (Hao et al., 2018) 


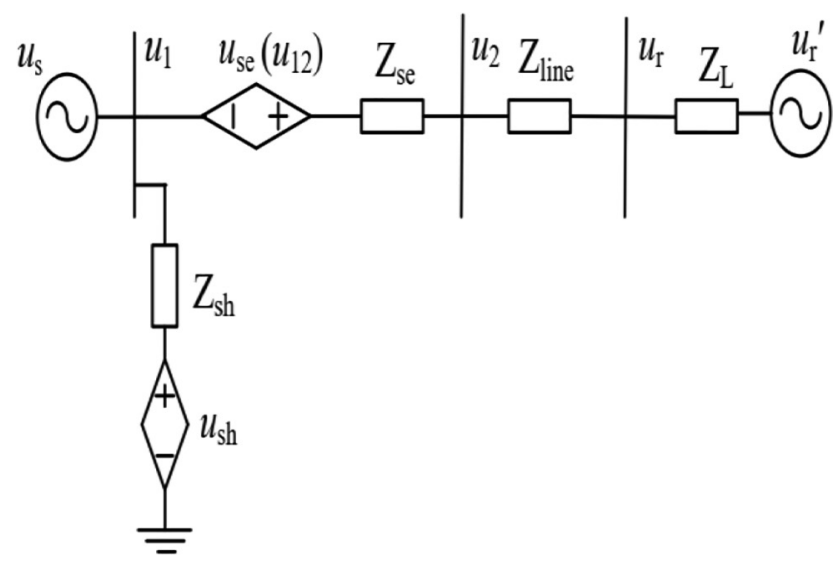

Fig. 3. The equivalent circuit of modular multilevel converter-based unified power flow controller

In the case of unbalanced grid condition, MMC can be divided into positive- and negative-sequence subsystem. In Fig. 3, $U_{1}$ is the ac bus voltage, $Z_{\text {line }}$ is the unbalanced impedance, $Z_{\mathrm{L}}$ is the asymmetric load impedance, and $u_{\mathrm{r}}$ is the unbalanced ac bus voltage at the receiving end. When ac fault occurs, $u_{\mathrm{r}}$, it would become unbalanced.

The general control principle is to compensate the unbalanced components of $u_{\mathrm{r}}$ or $Z_{\text {line }}$ by regulating $u_{\mathrm{se}}\left(u_{12}\right)$ coupled from the series MMC, and the control objective is to obtain balanced ac currents of the transmission line by suppressing the negative-sequence current (NSC) and zero-sequence current (ZSC). In detail, the NSC and ZSC can be suppressed by injecting the corresponding zero-sequence voltage (ZSV) and the negative-sequence voltage (NSV) in usage.

\section{Problem formulation and design constraints}

The basic principle behind the problem of optimal power flow (OPF) is to minimise the production cost of the generator in terms of power balance and line flow constraints. The OPF problem is expressed as below:

$$
\text { Minimise } C=\sum_{i=1}^{N_{g}} F_{g i}
$$

Subject to equality constraints:

$$
P_{g i}=P_{d t}+\sum_{j=1}^{N}\left|V_{i}\right|\left|V_{j}\right|\left(G_{i j} \cos \delta_{i j}+B_{i j} \sin \delta_{i j}\right) \quad \forall i=1,2, \cdots, N
$$

$P_{d t}$ is signified as the demand of real power in $t^{\text {th }}$ bus, total buses are $N$, and $V_{i}$ and $V_{j}$ are the $i^{\text {th }}$ and $j^{\text {th }}$ node voltage magnitude $G_{i j}$ is the conductance of the line $i$ to $j$, and $B_{i j}$ is the susceptance of the line $i$ to $j$.

The $i j^{\text {th }}$ bus voltage is represented as $\delta_{i j}=\delta_{j}-\delta_{i}$.

Power flow constraints:

$$
\left|S l_{i j}\right| \leq S l_{i j}^{\max } \quad \forall i j \in N_{l}
$$

$S l_{i j}$ and $S l_{i j}^{\max }$ are the line $i$ to $j$ power flow and its maximum limit, respectively, and $N_{l}$ is the total lines.

Other in-equality constraints:

$$
\begin{aligned}
& P_{g i}^{\min } \leq P_{g i} \leq P_{g i}^{\max } \quad \forall i=1,2, \cdots, N_{g} \\
& \delta_{i}^{\min } \leq \delta_{i} \leq \delta_{i}^{\max } \quad \forall i=1,2, \cdots, N
\end{aligned}
$$




$$
v_{i}^{\min } \leq v_{i} \leq v_{i}^{\max } \quad \forall i=1,2, \cdots, N
$$

The fuel costs of generator $i$ can be mathematically represented by:

$$
F_{g i}=\frac{1}{2} a_{g i} P_{g i}^{2}+b_{g i} P_{g i}+c_{g i}(\$ / \mathrm{h})
$$

$F_{g i}$ is the total fuel cost $(\$ / \mathrm{h})$, and the cost coefficients of the distributed generator are given as $a_{g i}, b_{g i}$, and $c_{g i} . P_{g i}$ is the $i^{\text {th }}$ generator active power.

\section{DGs optimal sizing}

In order to obtain the optimal capacities of DG units, the OPF-based transmission control management (TCM) problem is considered in the present work. In this regard, a novel objective function comprising of voltage improvement (VI) factors, line flows reduction are considered as technical parameters and real power losses (RPLs), as well as DG costs, are considered as the economic parameters.

Minimisation of six objectives is the main aim of the proposed multiobjective IPSO optimisation algorithm.

Total congestion cost (TCC): Initially the OPF is executed to find the TCC. Based on the OPF results, the flow of each line and locational marginal prices (LMPs) are calculated. The TCC can be computed as follows:

$$
\text { Minimise TCC }=w_{1} \times \sum_{i j=1}^{N_{l}} \Delta \rho_{i j} \times P_{i j}
$$

Where $P_{i j}$ is the power flow of line $i, j, N_{l}$ is the number of transmission lines, and $w$ is the scaling factor.

$$
\Delta \rho_{i j}=\left|L M P_{i}-L M P_{j}\right|
$$

Voltage violation (VV): $\mathrm{V}$ is calculated based on the summation of the magnitude of bud voltage and penalty factor (PF) for bus voltage.

$\mathrm{V}$ is defined as:

$$
\text { Minimise VV }=w_{2} \times \sum_{i=1}^{N}\left(P F \times\left(V_{i}-1\right)^{2}\right)
$$

Where $V_{i}$ is the magnitude of bud voltage $I$, and $N$ is the number of buses. The PF for bus voltage is described as:

$$
\mathrm{PF}=\left\{\begin{array}{ll}
1 & 0.95 \leq V_{i} \leq 1.05 \\
5 e^{\left(V_{i}-1.05\right)} & V_{i} \geq 1.05 \\
5 e^{\left(0.95-V_{i}\right)} & V_{i} \leq 0.95
\end{array}\right\}
$$

VI: The fraction that describes the relationship in-between the sum of squares of the deviations of bus voltage from 1 p.u. before and after incorporation of the DGs is defined as VI.

$$
\text { Minimise VI }=w_{3} \times \frac{\sum_{i=1}^{N}\left(V_{i}^{D G}-1.0\right)^{2}}{\sum_{i=1}^{N}\left(V_{i}^{0}-1.0\right)^{2}}
$$

$V_{i}^{D G}$ is the $i^{\text {th }}$ node voltage magnitude after insertion of DG and $V_{i}^{0}$ is the $i^{\text {th }}$ node voltage magnitude after insertion of DG. 
RPLs: The ratio of actual power losses after and before DGs in the network of the power system is considered as RPL.

It is formulated as:

$$
\text { Minimise RPL }=w_{4} \times \frac{P_{\mathrm{Loss}}^{\mathrm{DG}}}{P_{\mathrm{Loss}}^{0}}
$$

$P_{\text {Loss }}^{\mathrm{DG}}$ and $P_{\text {Loss }}^{0}$ are the RPLs before and after including DG.

Cost factor (CF): The objective is used to achieve the utmost investment in favourable costs of conventional generators and DGs. It is defined as:

$$
\text { Minimise } \mathrm{CF}=\sum_{i=1}^{N_{g}} F_{g i}+w_{5} \times \frac{\sum_{i=1}^{N_{\mathrm{DG}}} F_{\mathrm{DG}, i}}{P_{\mathrm{DG}}^{\max }}
$$

$P_{\mathrm{DG}}^{\max }$ is the $k^{\text {th }} \mathrm{DG}$ maximum power penetration, $F_{g i}$ is the fuel costs of generator $i$ and $F_{D G, i}$ is the fuel costs of DGs.

Transmission congestion (TC): The ratio of the active flow of power in each of the transmission corridors after the insertion of the DG(s), and its maximum limit is evaluated for all the corridors of the whole network. The fractions of the maximum value are known to be a congestion transmission factor. It is mathematically formulated as:

$$
\text { Minimise TC }=w_{6} \times \operatorname{maximum}\left(\frac{s l_{i j}^{D G}}{S l_{i j}^{\max }}\right)
$$

$S l_{i j}$ and $S l_{i j}^{\max }$ is the line $i$ to $j$ power flow and its maximum limit, respectively.

The strategy of the weighting factor is used to transform the parameters of technical and economic into a single objective function to attain the DG optimal capability units. The multiobjective function solutions lack of weighting strategy can deviate to conflicting solutions. Thus, in this work, normalised weights are used to form the final fitness function to be optimised to achieve optimal capabilities. The weighted multigoal fitness function is shown below:

$$
J=h_{1} \times T C C+h_{2} \times V V+h_{3} \times V I+h_{4} \times R P L+h_{5} \times C F+h_{6} \times T C
$$

The fitness function (Equation 16) is minimised based on power balance constraints and other inequality constraints. Owing to the effect of DGs, real power balance constraint (2) is changed as follows:

$$
\begin{gathered}
P_{g i}=P_{d t}+\sum_{j=1}^{N}\left|V_{i}\right|\left|V_{j}\right|\left(G_{i j} \cos \delta_{i j}+B_{i j} \sin \delta_{i j}\right) \forall i=1,2, \cdots, N \\
P_{g i}+P_{D G, k}=P_{d t}+\sum_{j=1}^{N}\left|V_{i}\right|\left|V_{j}\right|\left(G_{i j} \cos \delta_{i j}+B_{i j} \sin \delta_{i j}\right) i=k
\end{gathered}
$$

Owing to the flexible operation of DGs, they can be integrated for test bus systems for both long-term and shortterm planning horizons. In the long-term planning horizons, they are integrated into the distribution to improve the economic and technical performance of the system. In the short-term planning horizons, DGs are identical to negative energy injections (bus-PV) which, if maintained in optimal position, reduce the net load in that bus by reducing the flow of real power in the lines during the Newton Raphson (NR) load flow analysis. The finite amount of works used the insertion of the DG on transmission networks as the short-term horizon to mitigate in the network congestion lines. 


\section{Multiobjective IPSO for congestion and voltage profile management}

The production cost function of the generator is minimised by power balancing constraints and the basic problem of the line flow constraints in the OPF.

\section{a) PSO}

The PSO an evolutionary optimisation was modelled based on the social behaviour of birds who flock or feed on fish. This algorithm contains a population that continuously updates the knowledge of the search space. This population is made up of individuals, each of which signifies a possible solution and modelled as a moving particle through hyperspace. Each position and the speed of particles are updated as:

$$
\begin{aligned}
& V_{i}^{k+1}=w^{k} \times V_{i}^{k}+C_{1} \times \operatorname{rand}()_{1} \times\left(p_{i}^{k}-X_{i}^{k}\right)+C_{2} \times \operatorname{rand}()_{2} \times\left(g_{\text {best }}^{k}-X_{i}^{k}\right) \\
& X_{i}^{k+1}=X_{i}^{k}+\text { velocity }_{i}^{k+1}
\end{aligned}
$$

where $V_{i}^{k}$ is the particle $i^{\text {th }}$ velocity at the $k^{\text {th }}$ iteration, $V_{i}^{k+1}$ is the velocity of the $i^{\text {th }}$ particle at $(k+1)$ the iteration, $w^{k}$ is an inertia weight iteration of $k^{\text {th }}$, and $\operatorname{rand}()_{1}$ and $\operatorname{rand}()_{2}$ are the random numbers selected in-between 0 's and 1's. The inertial weight allows controlling the swarm of the exploration and the exploitation in addition to controlling the speed and direction of the particles.

$C 1$ and $C 2$ are positive coefficients between 0 and 2 that are $C 1+C 2 \leq 4$. The original PSO frequently suffers from the trapping problem in the local optima to meet prematurely. The performance of the original PSO is very dependent on $C 1, C 2$, and $w^{\mathrm{k}}$.

Most of the improved algorithms are improved by adjusting the parameters of the algorithm, such as introducing various linear and nonlinear inertial weight dynamic adjustment strategies, and introducing contraction factors and so on. The proposed particle swarm improvement algorithm has improved performance and the efficiency, yet it needs large improvement, and so to attain a particle swarm algorithm with better performance, higher efficiency, and lower costs, the Academic and industry researchers have explored and tried out new ways to improve.

In order to avoid this disadvantage, this paper proposes an improved particle swarm optimisation (IPSO) with chaos adaptive algorithm to compute the inertia weight $(\mathrm{v})$ and a self-adaptive method to compute learning factors $\left(C_{1}\right.$ and $\left.C_{2}\right)$. The algorithm evolves the inertia weight through adaptive adjustment strategy and optimises the learning factor by the chaotic sequence generated by chaos theory. The IPSO can improve the algorithm's precocious problem and improve the searching speed of the algorithm. Finally, the algorithm was applied for resolving multiobjective optimisation problems to verify the accuracy performance of convergence, speed, global convergence, and so on.

\section{b) Fuzzy logic control (FLC)}

Fuzzy logic control is an ill-defined process of complex function managed by a trained human without significant system dynamics. In an FLC, the initial idea is to use the expert operator knowledge for the construction of FLC, which performs the system control; then, the input-output variables of the system were characterised by fuzzy rules (IF-THEN) to the difference with a mathematical complicated model. In the FLC design, the linguistic variables were used by fuzzy rules and the reasoning of fuzzy allows the incorporation of the human expert experience.

\section{c) IPSO}

Numerous works have been developed to dynamically adjust the inertial weight $w^{\mathrm{k}}$. We propose adjusting the inertia weight $w^{\mathrm{k}}$ and learning factors $C_{1}$ and $C_{2}$ of the PSO during the iteration process. The factors of learning are $C_{1}$ and 
$C_{2}$ that influence the total velocity of a particle. The cognitive component $C_{1}$ determines its own confidence in the particle, and the social component $C_{2}$ defines the confidence of the particle in the neighbours. To design a blurred inference system for the adaptation of inertial weight and learning factors, the design consists of the following:

Two inputs: the number of iterations (NU) when the best fitness does not change the actual value of the inertial weight $\left(w^{k}\right)$.

Three outputs: the change in inertia weight $\left(c h w^{k}\right)$ and the change in learning factors $C_{1}$ and $C_{2}$. For each input and output fuzzy interference system, the three membership functions are explained as HIGH, LOW, and MEDIUM, respectively, represented as the left triangle, the central triangle, and the right triangle.

To obtain the new $c h w^{\mathrm{k}}, C_{1}$ and $C_{2}$ values, nine fuzzy rules are used in the fuzzy inference system. An example of one fuzzy rule is given as follows:

If NU is MEDIUM, and

$$
w^{\mathrm{k}} \text { is } \mathrm{HIGH}
$$

then $C_{1}$ is $\mathrm{HIGH}, C_{2}$ is $\mathrm{HIGH}$, and $c h w^{\mathrm{k}}$ is LOW.

The mathematical descriptions for $w^{\mathrm{k}}$ is defined with the following expressions:

$$
w^{k}=w_{\max }-\left(w_{\max }-w_{\min }\right)\left\{\frac{\left(\text { iter }_{\max }-\text { iter }\right)^{n}}{\left(\text { iter }_{\max }\right)^{n}}\right\}
$$

Where iter is the number of current iteration of the algorithm, iter ${ }_{\text {max }}$ is the maximum number of iterations that the PSO algorithm can execute, and $n$ is a modulation of the nonlinear index.

The mathematical descriptions for $C_{1}$ and $C_{2}$ are defined with the following expressions:

$$
C_{1}=\frac{\sum_{i=1}^{r_{C_{1}}} \mu_{i}^{C_{1}}\left(c_{1 i}\right)}{\sum_{i=1}^{r_{c_{1}}} \mu_{i}^{C_{1}}}
$$

Where $C_{1}$ is the percentage of cognitive acceleration of the particle $i, r_{c 1}$ is a number of fuzzy rules activated to $C_{1}, c_{1 i}$ is the output of the fuzzy rule $i$ for $C_{1}$, and $\mu_{i}^{C_{1}}$ is the membership function value of fuzzy rule $i$ for $C_{1}$.

$$
C_{2}=\frac{\sum_{i=1}^{r_{C 2}} \mu_{i}^{C_{2}}\left(c_{2 i}\right)}{\sum_{i=1}^{r_{c_{1}}} \mu_{i}^{C_{2}}}
$$

Where $C_{2}$ is the percentage of cognitive acceleration of the particle $i, r_{c 2}$ is a number of fuzzy rules activated in $C_{2}, c_{2 i}$ is an output of the fuzzy rule $i$ for $C_{2}$, and $\mu_{i}^{C_{2}}$ is the membership function value of the fuzzy $i$ rule for $C_{2}$.

Flowchart to achieve the best possible capabilities of DG units with the proposed IPSO algorithm is shown in Fig. 4. First read the system data, then solve OPF problem without DG placement. After that calculate the LMPs and the power flows of all lines in the system. Then, assign the weight factors to the objective function. The IPSO is used to find the optimal values of the MMC-UPSC and the appropriate location. The calculation is mainly based on the fitness function. In IPSO, the parameters are initialised first. The procedure is the same as that of the standard PSO but updating the velocity based on the FLC. It also performs adjusting the inertia weight $w^{\mathrm{k}}$ and $C_{1}, C_{2}$ are factors of learning during the iteration process. 


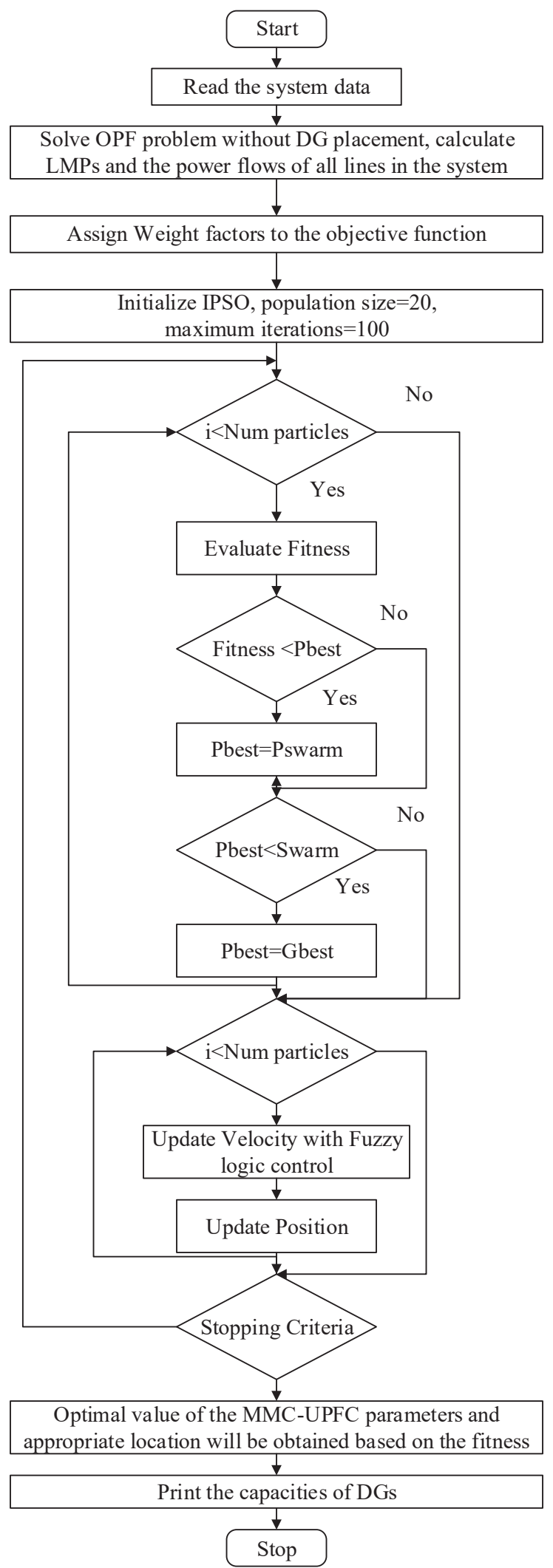

Fig. 4. Basic flow chart of the proposed approach to the management of congestion by improved particle swarm optimisation 


\section{Experimental result and analysis}

The efficiency of the novel approach is checked on the 30-bus and 118 IEEE-bus test systems using MATLAB. The convergence plot for the fitness and voltage profile of the two bus systems are evaluated to show the performance. The experimental results of the proposed approach are evaluated using MATLAB, and it is tested under two standard bus test systems, that is, IEEE - 30 buses and 118 buses. The fitness and voltage profile are evaluated for the two bus systems, and their performance has been presented using the convergence plot. A comparative analysis is performed with the conventional techniques to check and validate the performance of the proposed system. The below sections illustrate the comparative analysis by two cases based on the two test system.

\subsection{Case 1: IEEE 30 bus}

Initially, the IEEE 30-bus system is used to test the proposed methodology. The 30-bus system in IEEE consists of 21 loads, the 41 transmission lines, and 6 generators (Singh et al., 2014). The details related to the buses (upper and lower limits of generation of real energy and emission coefficients of the generators) and the network lines are taken from the reference (Panigrahi and Ravikumar Pandi, 2009).

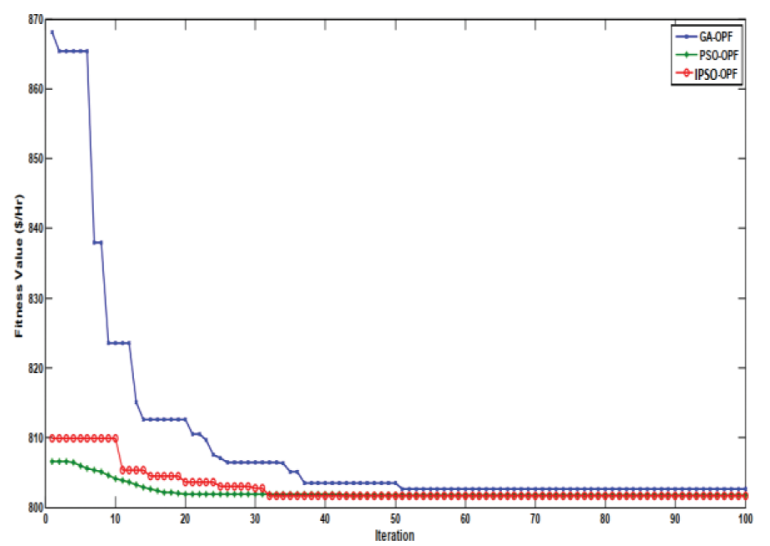

Fig. 5. Curves convergence for system 1 during optimal power flow (OPF) run

The convergence curve for the OPF performed in the case of the system 1 with all three algorithms reported is shown in Fig. 5.

a)

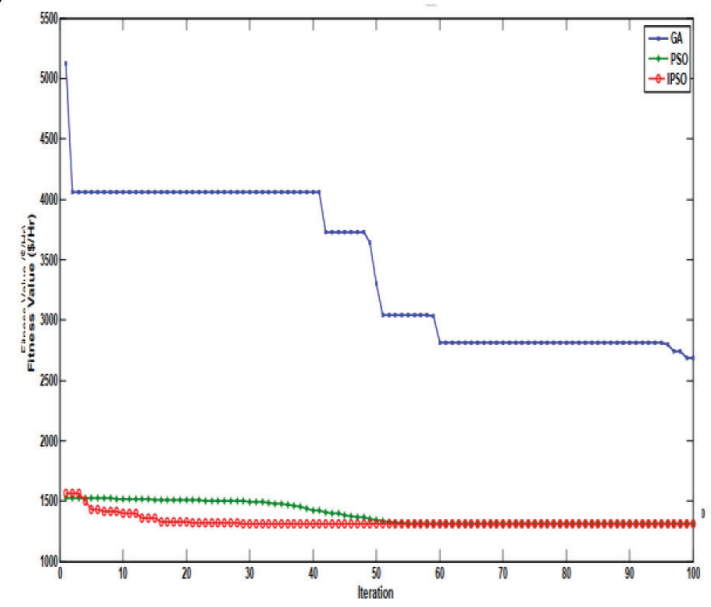

b)

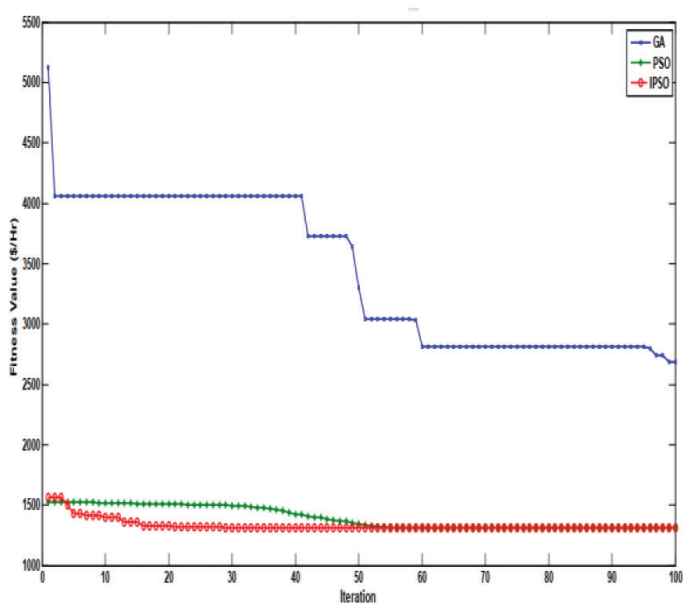

Fig. 6. (a) Convergence curves for system during case $1 \mathrm{C}$ by using modular multilevel converter-based unified power flow controller distributed generation (DG). (b) Convergence curves for system during case $1 \mathrm{C}$ by using 0.9 lagging power factor DG 
Convergence features show stability, final fitness value, and the number of iterations adopted to converge a particular algorithm. It is desirable that algorithms with high convergence rates are better than the algorithms with low convergence rates. In this outlook, the curves of convergence through the run of $1 \mathrm{C}$ case considering the delayed power factors UPF and 0.9 by the methods IPSO, PSO, and the GA are shown in Fig. $6(\mathrm{a})$ and (b), respectively.

The above figures show that the IPSO approach converges in fewer iterations, which achieves a low value of the fitness function in contrast to the PSO and GA approaches. This shows the superiority of the proposed IPSO algorithm over the other reported approaches.

a)

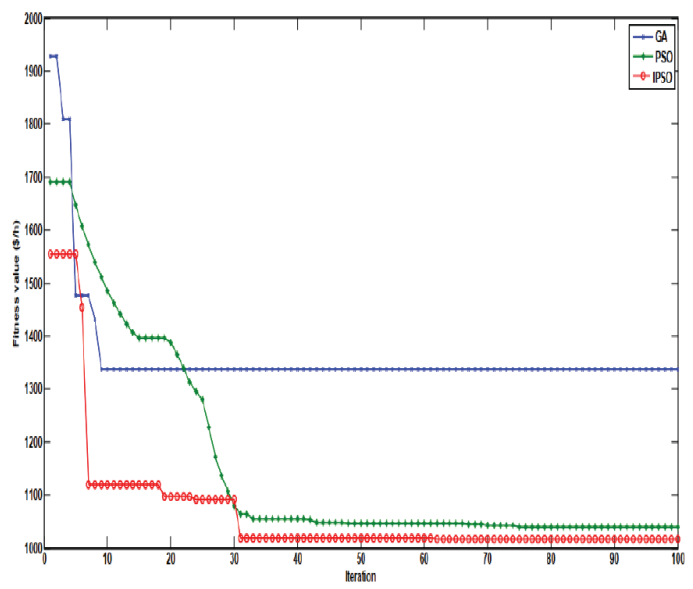

b)

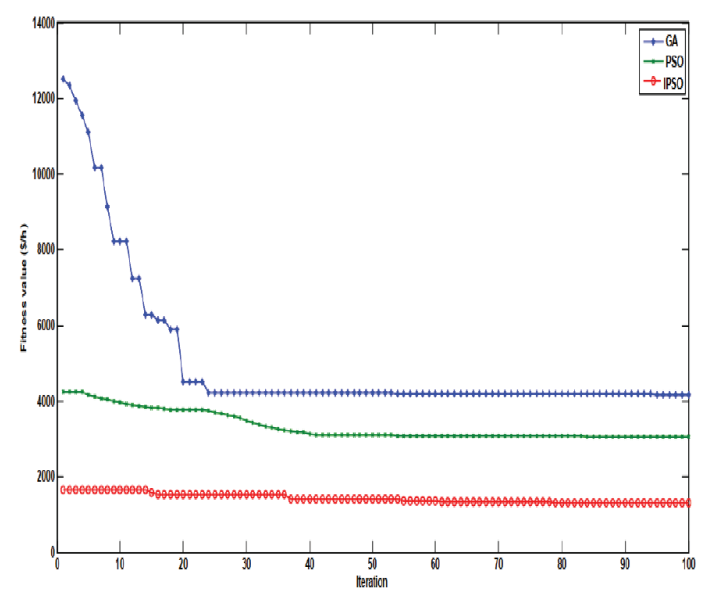

Fig. 7. (a) Convergence curve for the system-Case 1D by using modular multilevel converter-based unified power flow controller distributed generation (DG). (b) Convergence curve for the system-Case 1D by using 0.9 lagging power factor DG

Likewise, the convergence curves of the algorithms reported during the execution of the 1D case for both the delayed power factor DG UPF and 0.9 are revealed in Fig. 7(a) and (b), respectively.

From the figures, it can be revealed that the convergence characteristics of the proposed IPSO approach are further improved as compared to the case $1 \mathrm{C}$ with fine tuning of the objective function.

\subsection{Case 2: IEEE - 118 bus}

The insisted method is practiced on system 2 that consists of 186 transmission lines, 99 loads, and 54 generators. The real power demand on the system 2 is 4,242 MW. The lower and upper limits of real power generation, bus data, line data, and the emission coefficients of the generators related to system 2 can be accessed from the study by Pratap et al. (2016). Leaving the generator on bus 69 as a reference, the generator outputs excluding the 69th bus generator are considered as variable quantities for the base OPF run. The basic results of OPF obtained using IPSO were compared with the several works available in the previous literature.

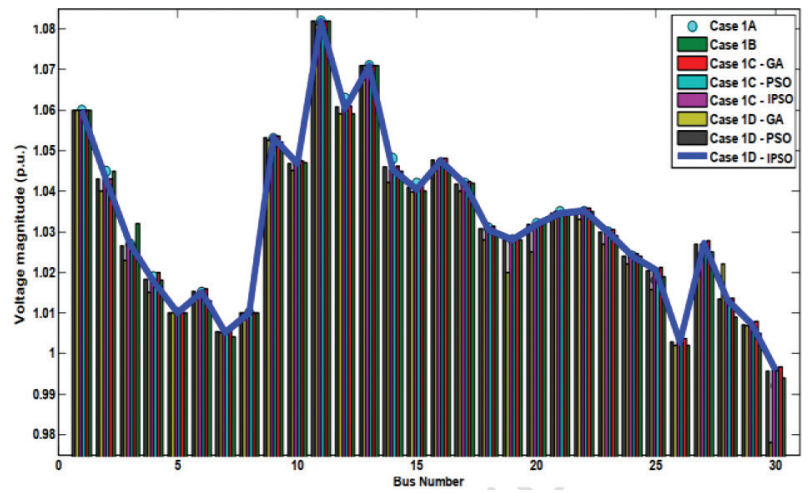

Fig. 8. Voltage profile of system 1 when considering modular multilevel converter-based unified power flow controller distributed generation 
Fig. 8 shows the bus voltage profile in p.u. with and without the DG UPF for both $1 \mathrm{C}$ and $1 \mathrm{D}$ cases. Likewise, Fig. 9 shows the bus voltage profile with and without 0.9 delay factors. From the previous figures, it has been clarified that the improvement of the voltage profile is greater after having connected the optimal capacities of the DGs operating to a power factor delayed of 0.9 with respect to the UPF. The figure also reveals the progress of the IPSO algorithm compared to other approaches.

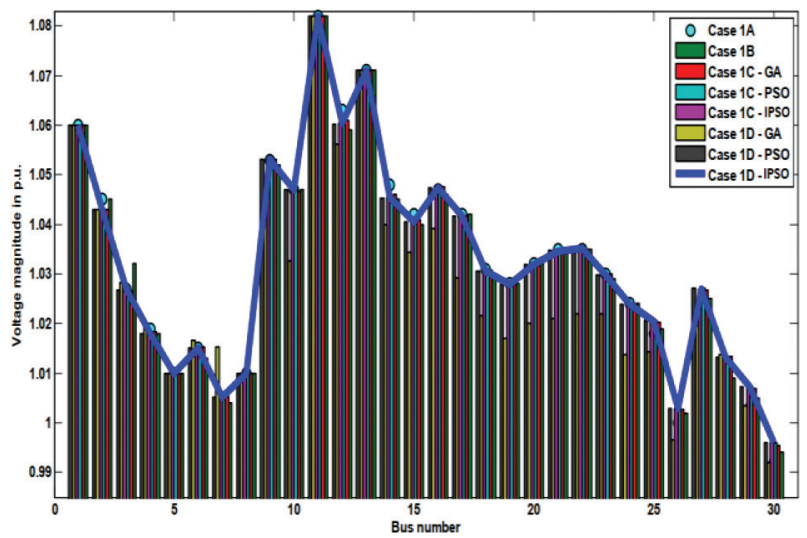

Fig. 9. Voltage profile of system 1 when considering 0.9 lagging power factor distributed generations

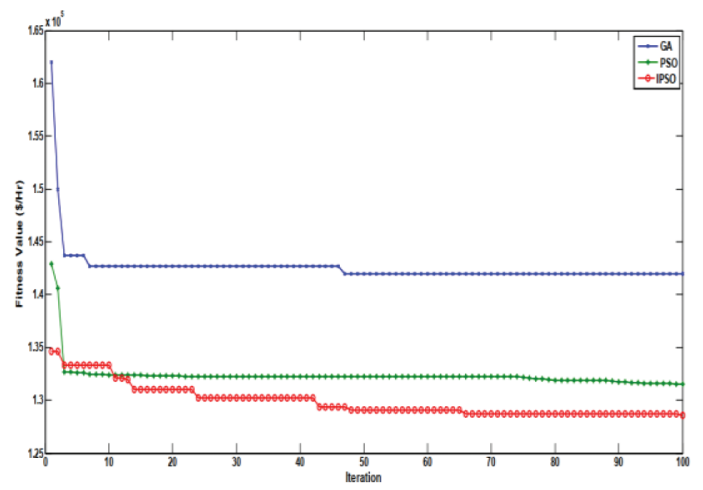

Fig. 10. Convergence curve for the optimal power flow run of IEEE 118-Bus system

The convergence curve for the basic execution of OPF in the case of a 118-Bus system in IEEE has all the algorithms reported is shown in Fig. 10.

a)

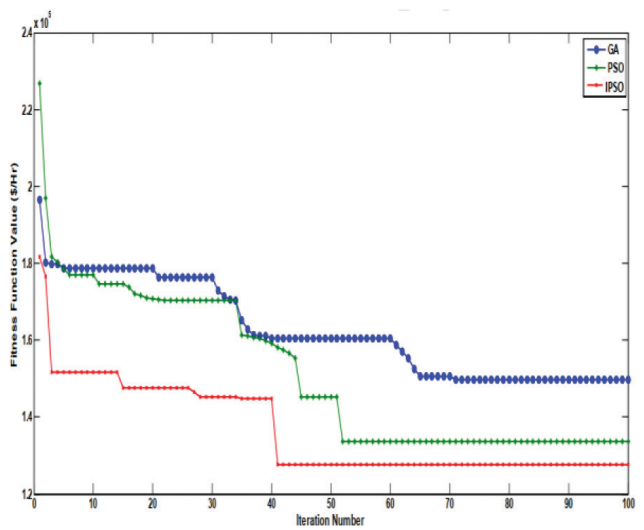

b)

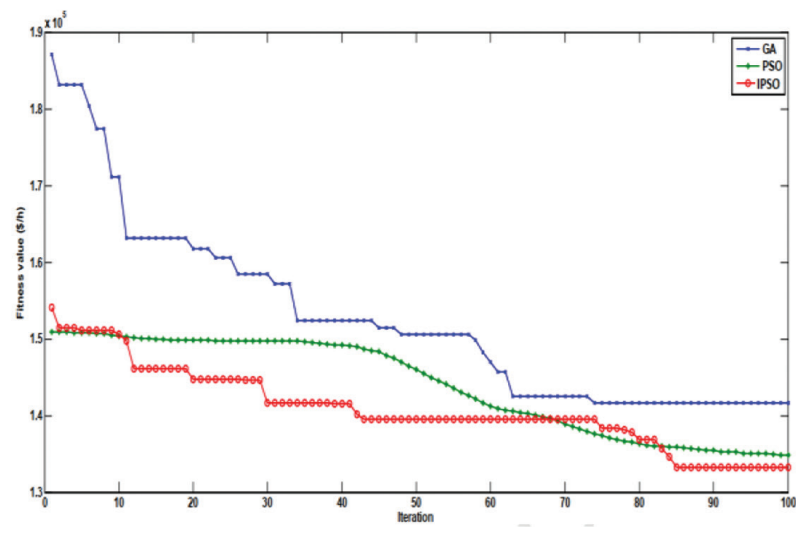

Fig. 11. (a) Convergence curve for the system - Case $2 \mathrm{C}$ by using modular multilevel converter-based unified power flow controller distributed generation (DG). (b) Convergence curve for system - Case 2C by using 0.9 lagging power factor DG 
Here also, the convergence graphs of the proposed IPSO and other reported algorithms for the case 2C considering the UPF and 0.9 lagging power factor DGs are shown in Fig. 11(a) and (b), respectively.

a)

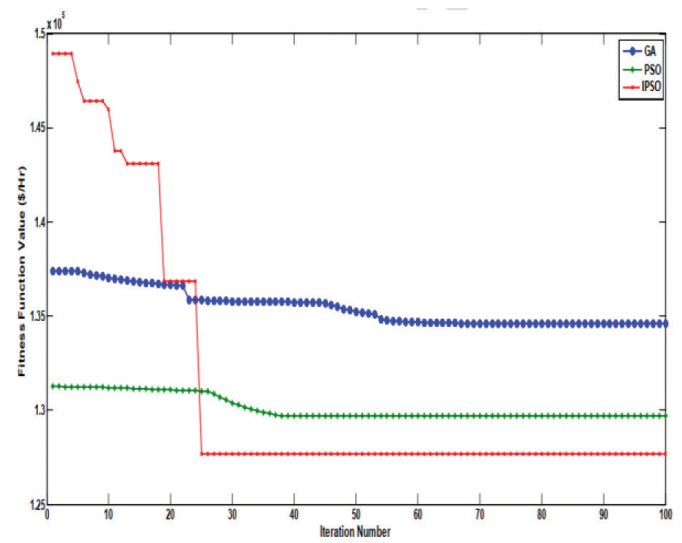

b)

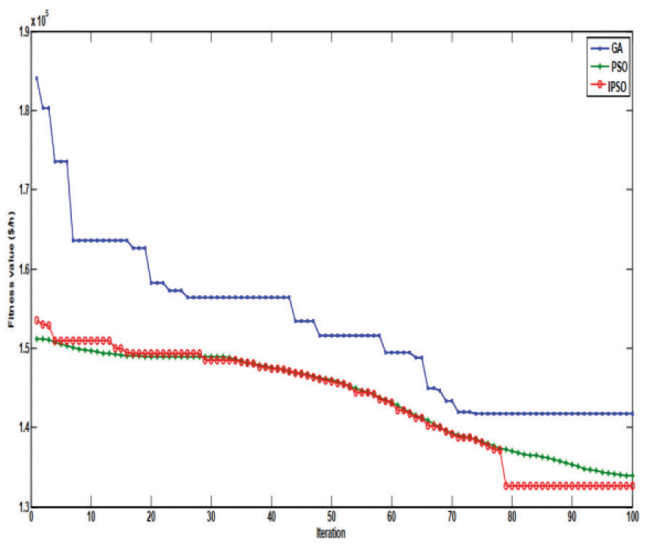

Fig. 12. (a) Convergence curve for system - Case $2 \mathrm{D}$ by using modular multilevel converter-based unified power flow controller distributed generation (DG). (b) Convergence curve for the system - Case 2D by using 0.9 lagging power factor DG

Correspondingly, the curves of convergence in the 2D case for the unit-delayed power factor DGs and the 0.9 from the stated algorithms are shown in Fig. 12(a) and (b), respectively.

It is clear from the figures that the convergence characteristics in the case $2 \mathrm{C}$ are moderate as compared to the case 2D. Overall, the convergence characteristics of the proposed IPSO approach are better as compared to the other reported algorithms.

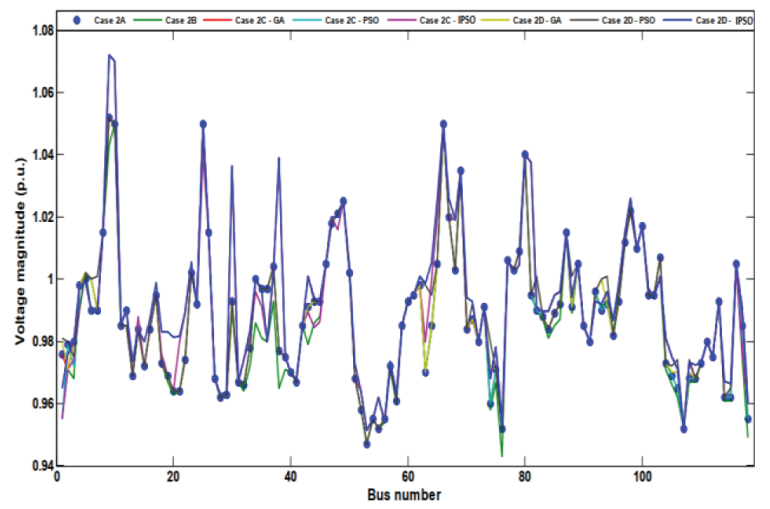

Fig. 13. Voltage profile of system 2 with optimal capacities of unity power factor distributed generation

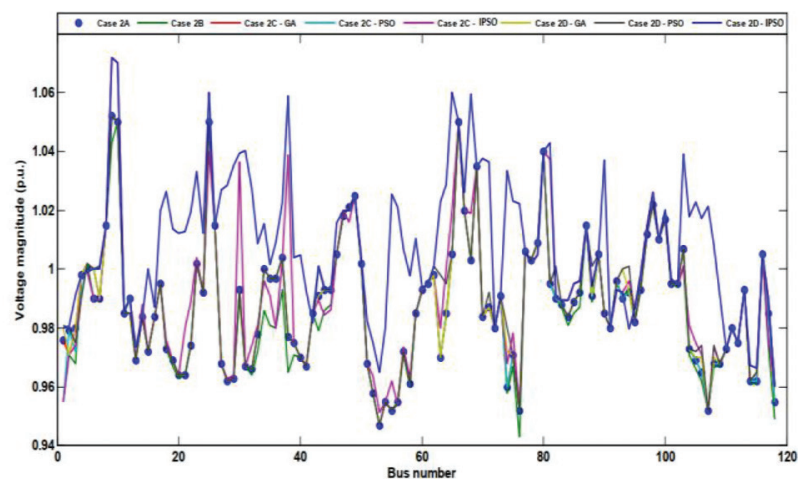

Fig. 14. Voltage profile of system 2 with optimal capacities of 0.9 lagging power factor distributed generation 
Fig. 13 shows the bus profile voltage in p.u. with and without DG UPF for both $2 \mathrm{C}$ and 2D cases. Similarly, Fig. 14 indicates the bus voltage profile with and without 0.9 delay factors. From the previous figures, it has been clarified that the improvement of the voltage profile is greater after having connected the optimal capacities of the DGs operating to a power factor delayed of 0.9 with respect to the UPF. The figure also reveals the progress of the IPSO algorithm compared to other approaches.

Concisely, the whole result reveals that congestion lines are completely calmed without any overloading of another network line of the mass power systems. In addition, the results also state the superiority of IPSO over PSO and GA in terms of convergence characteristics, real reduction in power loss, line flow limitation and flexibility when applied to both small and huge test bus systems. Benchmarking is performed to reveal that the proposed multiobjective approach and its solution methodology are the best alternatives to reduce energy flows in congested lines.

\section{Conclusion}

In this work, the management of optimal energy flow congestion is managed by incorporating optimal capacity of DG units to the mass-power system network. A new multiobjective function that includes both the technical and economic parameters of the network, which is made to attain the optimal capabilities of the DG units. An optimised IPSO is proposed to provide the best optimal solution for the proposed multiobjective function. Available DG technologies are able to operate between ranges from 0.95 to 0.95 in advance. In general, DGs operating in delayed power factors are able to supply reactive energy to the system which internally improves the voltage profile of the entire network. Therefore, results with MMC-UPFC and 0.9 delay factors in terms of power factor are also evaluated and compared. The methodology was performed on 30-Bus in IEEE and the 118-Bus standard systems to verify the proposed contributions effectiveness.

\section{Acknowledgments}

I would like to thank my supervisor, Dr Geetha Ramadas, Professor and Head of Department EEE, for her excellent guidance, where the initial scope of the thesis was defined, and throughout the process of writing this thesis. I would like to thank anonymous reviewers for their comments.

\section{References}

Albatsh, F. M., Mekhilef, S., Ahmad, S. and Mokhlis, H. (2017). Fuzzy-Logic-Based UPFC and Laboratory Prototype Validation for Dynamic Power Flow Control in Transmission Lines. IEEE Transactions on Industrial Electronics, 64(12), pp. 9538-9548.

Batra, I. and Ghosh, S. (2018). An Improved Tent MapAdaptive Chaotic Particle Swarm Optimization (ITM-CPSO)-Based Novel Approach Toward Security Constraint Optimal Congestion Management. Iranian Journal of Science and Technology, Transactions of Electrical Engineering, pp. 1-29.

Dutta, S. and Singh, S. P. (2008). Optimal Rescheduling of Generators for Congestion Management Based on Particle Swarm Optimization. IEEE Transactions on Power Systems, 23(4), pp. 1560-1569.
Guan, M. and Xu, Z. (2012). Modeling and Control of A Modular Multilevel Converter-Based HVDC System Under Unbalanced Grid Conditions. IEEE Transactions on Power Electronics, 27(2), pp. 4858-4867.

Gyugyi, L. (2003). Unified Power-Flow Control Concept for Flexible AC Transmission Systems Generation. Proceedings of Transmission Distribution Conference, 139, pp. 323-331.

Hajforoosh, S., Nabavi, S. M. and Masoum, M. A. (2012). Coordinated Aggregated-Based Particle Swarm Optimisation Algorithm for Congestion Management in Restructured Power Market by Placement and Sizing of Unified Power Flow Controller. IET Science, Measurement \& Technology, 6(4), pp. 267-278. 
Hao, Q., Man, J., Gao, F. and Guan, M. (2018). Voltage Limit Control of Modular Multilevel Converter Based Unified Power Flow Controller Under Unbalanced Grid Conditions. IEEE Transactions on Power Delivery, 33(3), pp. 1319-1327.

Jordehi, A. R. (2015). Particle Swarm Optimisation (PSO) for Allocation of FACTS Devices in Electric Transmission Systems: A Review. Renewable and Sustainable Energy Reviews, 52, pp. 1260-1267.

Kapse, S. S. S., Daigavane, M. B. and Daigavane, P. M. (2018). Improvement of ORPD Algorithm for Transmission Loss Minimization and Voltage Control Using UPFC by HGAPSO Approach. Journal of the Institution of Engineers (India): Series B, pp. 1-11.

Kumar, A. and Sekhar, C. (2013). Congestion Management with FACTS Devices in Deregulated Electricity Markets Ensuring Loadability Limit. International Journal of Electrical Power \& Energy Systems, 46, pp. 258-273.

Kumar, A., Srivastava, S. C. and Singh, S. N. (2005). Congestion Management in a Competitive Power Market: A Bibliographical Survey. Electric Power Systems Research, 76(1-3), pp. 153-164.

Mishra, A. and Kumar Gundavarapu, V. N. (2016). Line Utilisation Factor-Based Optimal Allocation of IPFC and Sizing Using Firefly Algorithm for Congestion Management. IET Generation Transmission and Distribution, 10(1), pp. 115-122.

Mukherjee, V. and Verma, S. (2016). Optimal Real Power Rescheduling of Generators for Congestion Management Using A Novel Ant Lion Optimiser. IET Generation Transmission and Distribution, 10(10), pp. 2548-2561.

Panigrahi, B. K. and Ravikumar Pandi, V. (2009). Congestion management using adaptive bacterial foraging algorithm. Energy Conversion and Management, 50(5), pp. 1202-1209.
Pillay, A., Karthikeyan, S. P. and Kothari, D. P. (2015). Congestion Management in Power Systems A Review. International Journal of Electrical Power \& Energy Systems, 70, pp. 83-90.

Pratap, R., Mukherjee, V. and Ghoshal, S. P. (2016). Particle Swarm Optimization with an Aging Leader and Challengers Algorithm for the Solution of Optimal Power Flow Problem. Applied Soft Computing Journal, 40, pp. 161-177.

Raj, S. and Bhattacharyya, B. (2018). Optimal Placement of TCSC and SVC for Reactive Power Planning Using Whale Optimization Algorithm. Swarm and Evolutionary Computation, 40, pp. 131-143.

Singh, K., Yadav, V. K., Padhy, N. P. and Sharma, J. (2014). Congestion Management Considering Optimal Placement of Distributed Generator in Deregulated Power System Networks. Electric Power Components and Systems, 42(1), pp. 13-22.

Tu, Q., Xu, Z. and Xu, L. (2011). Reduced SwitchingFrequency Modulation and Circulating Current Suppression for Modular Multilevel Converters. IEEE Transactions on Power Delivery, 26(3), pp. 2009-2017.

Venkaiah, C. and Vinod Kumar, D. M. (2011). Fuzzy Adaptive Bacterial Foraging Congestion Management Using Sensitivity Based Optimal Active Power Re-Scheduling of Generators. Applied Soft Computing Journal, 11(8), pp. 4921-4930.

Wang, J. and Peng, F. Z. (2004). Unified Power Flow Controller Using the Cascade Multilevel Inverter. IEEE Transactions on Power Electronics, 19(4), pp. 1077-1084.

Yamin, H. Y. and Shahidehpour, S. M. (2003). Transmission Congestion and Voltage Profile Management Coordination in Competitive Electricity Markets. International Journal of Electrical Power \& Energy Systems, 25(10), pp. 849-861. 